\title{
Possible Relations Between Epstein-Barr Virus Past Infection and Classic Multiple Sclerosis in Guilan, Iran
}

\author{
Hamidreza Honarmand ${ }^{1}$; Masoumeh Ahmadi Jalali Moghadam ${ }^{2, *}$; Hamidreza Hatamian ${ }^{3}$; \\ Ali Roudbary ${ }^{3}$ \\ ${ }^{1}$ Department of Microbiology, Faculty of Medicine, Guilan University of Medical Sciences, Rasht, IR Iran \\ ${ }_{3}^{2}$ Cellular and Molecular Research Center, Faculty of Medicine, Guilan University of Medical Sciences, Rasht, IR Iran \\ ${ }^{3}$ Department of Neurology, Poursina Hospital, Guilan University of Medical Sciences, Rasht, IR Iran \\ *Corresponding author: Masoumeh Ahmadi Jalali Moghadam, Cellular and Molecular Research Center, Faculty of Medicine, Guilan University of Medical Sciences, Rasht, IR Iran. Tel: \\ +98-9113435232, Fax:+98-1316690036, E-mail: Masoumehjalaly@gmail.com
}

Received: November 6, 2013; Revised: June 1, 2014; Accepted: June 8, 2014

\begin{abstract}
Background: Multiple sclerosis (MS) is a demyelinating condition affecting the central nervous system. Although the cause of this condition is unknown, patients with MS seem to have genetic vulnerability to certain environmental factors such as infection that could trigger this condition.

Objectives: We conducted this study to determine whether MS risk increases following primary infection with Epstein-Barr virus (EBV) and also to investigate any association between MS and seropositivity to anti-EBNA-1 IgG, anti-EBV-CA IgG, and anti-EBV-EA.

Patients and Methods: EBV infection was confirmed using the Enzyme-Linked Immunoassay in the patient $(n=46)$ and control $(n=46)$ groups via commercial assays (anti-EBNA-1 IgG, anti-EBV-CA IgG, and anti-EBV-EA kits). The data were analyzed by using three statistical tests (Pearson chi-square, Spearman rho correlation, and odds ratio).

Results: Seropositivity to anti-EBNA-1 IgG did not show a significant difference between the patient and control groups (92.9\% and $88.4 \%$, respectively), and nor was seropositivity to anti-EBV-CA IgG different between the two groups ( $95.2 \%$ and 95.3\%, consequently). The antiEBV-EA-D test was negative in all the patients and in $95.3 \%$ of the controls. Seropositivity to both anti-EBNA-1 and anti-EBV-CA indicating past infection did not show significant associations with the later development of MS (Pearson chi-square asymptotic significance [Asymp. Sig.] [2-sided $]=0.317$, Spearman's rho correlation test Sig. [2-sided $]=0.689$, odds ratio $=1.95)$.

Conclusions: Seropositivity to both EBNA1- IgG and EBV-CA- IgG did not show a causal association with MS. The findings of this study suggest that EBV past infection could not be a causative factor in the development of MS and a protective factor against classic MS.
\end{abstract}

Keywords: Epstein Barr Virus Infections; Autoimmunity; Multiple Sclerosis

\section{Background}

Classic multiple sclerosis (MS) is a chronic degenerative disease and demyelinating condition affecting mainly the central nervous system. Women are more likely than men to contract the disease and it is the most common neurologically disabling disease in young adults. The etiology and pathogenesis of MS are unknown, but environmental agents and genetic susceptibility are likely to be involved. Although genetic predisposition plays an important role in determining susceptibility, past studies have shown that environmental factors are equally important (1). Epstein-Barr virus (EBV) is a human DNA herpes virus infecting more than $90 \%$ of the world's population. Infection in early childhood is common and usually asymptomatic. EBV is the etiological agent of infectious mononucleosis. Furthermore, diverse malignancies such as Burkitt and Hodgkin lymphoma have been associated with EBV. More recently, a possible role for EBV has been suggested in chronic inflammatory/autoimmune diseas- es like rheumatoid arthritis, systemic lupus erythematosus, and MS (2).

Multiple sclerosis is currently regarded as a disease with a multifactorial etiology. EBV infection might be one possible factor in MS manifestation. Infectious mononucleosis has been shown to increase the risk of developing MS later in life (1). In several studies, EBV seroprevalence rates were higher in adult and pediatric MS patients than in controls (3-8). Moreover, EBV antibody titers and EBV-specific T-cells showed an increase in MS patients as compared to healthy individuals (9). However, there is still controversy as to whether EBV is a causative agent or an innocent bystander in the pathogenesis of MS. Considering this question, in the present study we sought to evaluate the immune system response to EBV proteins evident in the blood samples from individuals with classis MS compared with those who did not have classic MS.

Copyright (C) 2015, Ahvaz Jundishapur University of Medical Sciences. This is an open-access article distributed under the terms of the Creative Commons Attribution-NonCommercial 4.0 International License (http://creativecommons.org/licenses/by-nc/4.0/) which permits copy and redistribute the material just in noncommercial usages, provided the original work is properly cited. 


\section{Objectives}

We aimed to investigate whether the presence of a possible latent or active EBV infection in individuals with classic MS could play a role in the development of the disease. Classic MS is a debilitating disease that is more common after ages at which EBV is prevalent. If there is a causal relation between EBV past infection and classic MS, it may be possible to prevent the latter by controlling the former.

\section{Patients and Methods}

This cross-sectional prospective study, conducted over an 8-month period between April 2012 and December 2012, included all clinical samples both from MS patients who were under the supervision of The MS Society of the Guilan Province, Northern Iran, and from healthy blood donors (age- and gender-matched with the patients) who voluntarily submitted specimens to Pars Medical Laboratory, Rasht, Iran. MS in the patients had been previously diagnosed via magnetic resonance imaging (MRI) and the Evoked Potential Test. The MS patients recruited also met the McDonald criteria. Serum samples were collected by standard methods. All the specimens were stored at $-70^{\circ} \mathrm{C}$ until the experiment was performed. Some demographical and other required data were collected by filling a questionnaire consisting of age, gender, disease duration, number of crises, interferon intake duration, the Expanded Disability Status Scale (EDSS) score, MRI result, Evoked Potential Test result, and antiviral therapy type (if prescribed).

A group of 46 subjects with classic MS along with 46 healthy controls were examined with the serological test for the presence of antibodies against EBV immunodominant antigens (EBNA-1, EBV-CA, and EBV-EA-D). All patients who were residents of the area (more than 6 months), were at an age range of 10 - 50 years, had classic MS (not opticospinal MS) confirmed by MRI and Evoked Potential Test, and were under the supervision of The MS Society of Guilan were included in the study. The exclusion criterion was comprised of opticospinal MS or any other presentations. Serological tests were performed using commercial Enzyme-Linked Immunoassay (ELISA) assays (anti-EBNA-1 IgG, anti EBV-CA IgG, and anti EBV-Ea-D IgG kits, EUROIMMUN Company, Germany). All the tests were performed by a laboratory technician and reported to the first author so as to blind the investigation. All the stages of the test were carried in accordance with the instructions of the company. Titers $>100 \mathrm{Ru} /$ $\mathrm{mL}$ were considered as high, titers from 51 to $100 \mathrm{Ru} / \mathrm{mL}$ were considered as medium, and titers from 22 to $50 \mathrm{Ru} /$ $\mathrm{mL}$ were considered as low. Seropositivity to both antiEBNA-1 IgG and anti-EBV-CA was interpreted as past infection and seropositivity to anti-EBV-EA-D was regarded as persistent infection.

The Statistical Package for Social Sciences (SPSS Inc.,
Chicago, IL, USA) (version 16) was used for the statistical analyses. The Pearson chi-square, Spearman rho correlation, and odds ratio tests were applied to analyze all the variables. All P values were regarded 2-tailed and significant at $\mathrm{P}<0.05$. The study conformed to the Helsinki Declaration and was reviewed and approved by the local research committee and ethics committee. Additionally, written informed consent was obtained from all the members of both groups.

\section{Results}

The average age of the patients $(n=46)$ was 32.6 years with a min-max of $13-51$. The age range of $42.8 \%$ of the patients was distributed between 28 and 38 years. The duration of the disease was more frequent between 2 and 9 years (76.3\%) (Figure 1). Most patients were women (71.4\%). The EDSS of most patients was between 2.5 and 3.5 (45.2\%), and $28.6 \%$ of the study population had an EDSS $<2.5$ (Figure 2). The Evoked Potential Test and MRI were positive in all the patients. Most patients (57.2\%) had a history of 1-2 crises of the disease (Figure 3 ).

Seropositivity to anti-EBNA-1 IgG was different between the patient and control groups (92.9\% and $88.4 \%$, correspondingly) (Figure 4), but this difference was not significant and did not indicate a causative association between seropositivity to anti-EBNA-1 IgG and the development of the disease (Table 1). Seropositivity to anti-EBV-CA IgG was not different between the patient and control groups (95.2\% and 95.3\%, respectively) (Figure 5). There was no significant association between anti-EBV-CA IgG and the development of MS (Table 1 ).

The anti-EBV-EA-D test was negative in all the patients, whereas it was positive in $4.7 \%$ of the controls, which can be interpreted as an indicator of persistent or reactivated EBV infection because those individuals also were seropositive to both anti-EBNA-1 IgG and anti-EBV-CA IgG. There was no significant association between anti-EBVEA-D IgG and the development of the disease (Table 1). Seropositivity to both anti-EBNA-1 IgG and anti-EBV-CA indicating past infection did not yield a significant correlation with the development of MS (Table 1). Finally, the other studied parameters did not show a significant association with seropositivity to anti-EBNA-1 IgG and antiEBV-CA IgG (Table 2).

\section{Discussion}

There is considerable evidence that EBV infection is a strong risk factor for the development of MS. Primary EBV infection at an early age is typically asymptomatic, but primary infection during adolescence or adulthood often manifests as infectious mononucleosis, which has been associated with a two- to threefold increased risk of MS (8). Most importantly, MS risk is extremely low in individuals who are EBV negative, but it increases considerably following EBV infection (10). Ruprecht et al. 


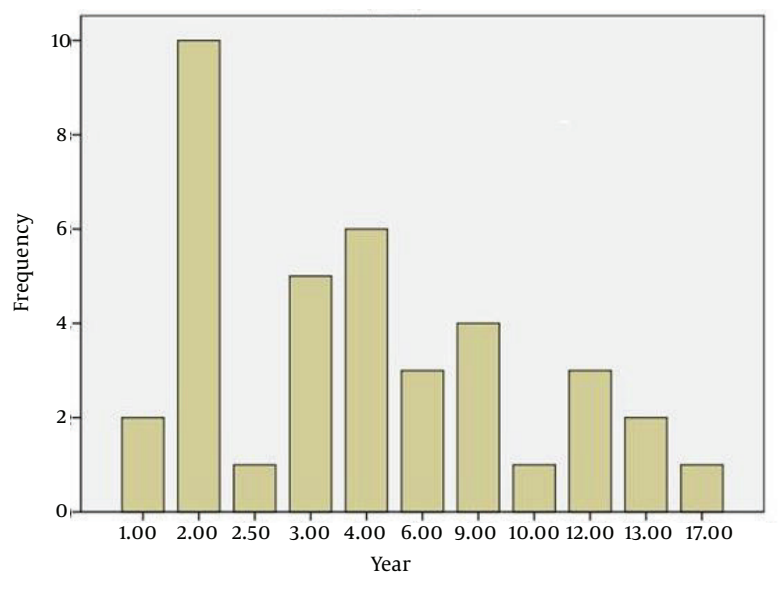

Figure 1. Frequency of the Duration of the Disease in the Patients

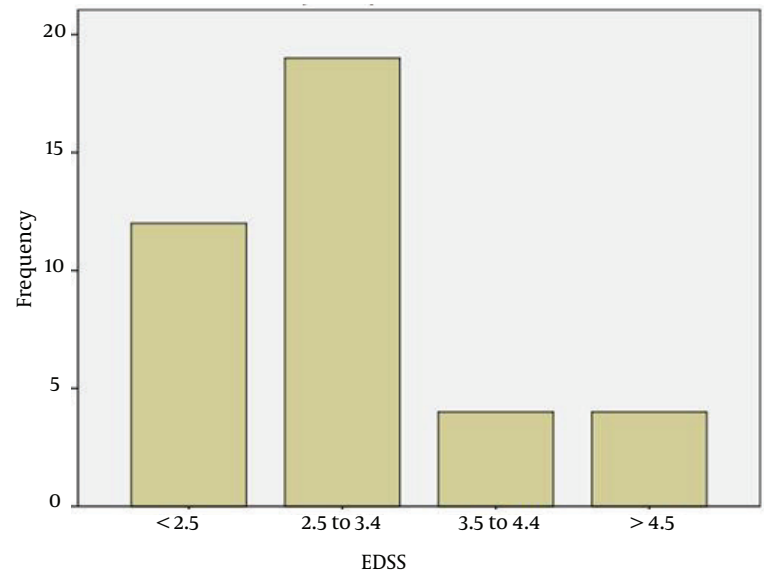

Figure 2. Distribution of the Expanded Disability Status Scale Score in the Patients

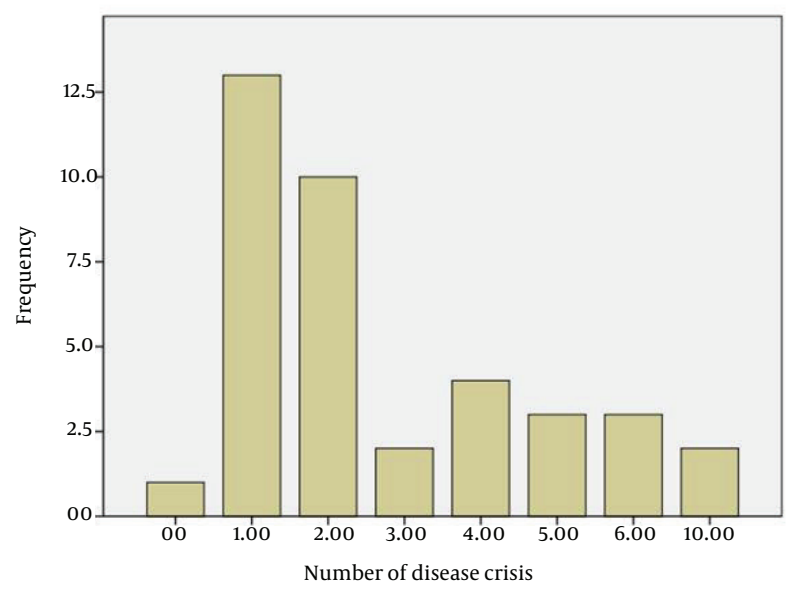

Figure 3. Frequency of the Disease Crisis in the Patients

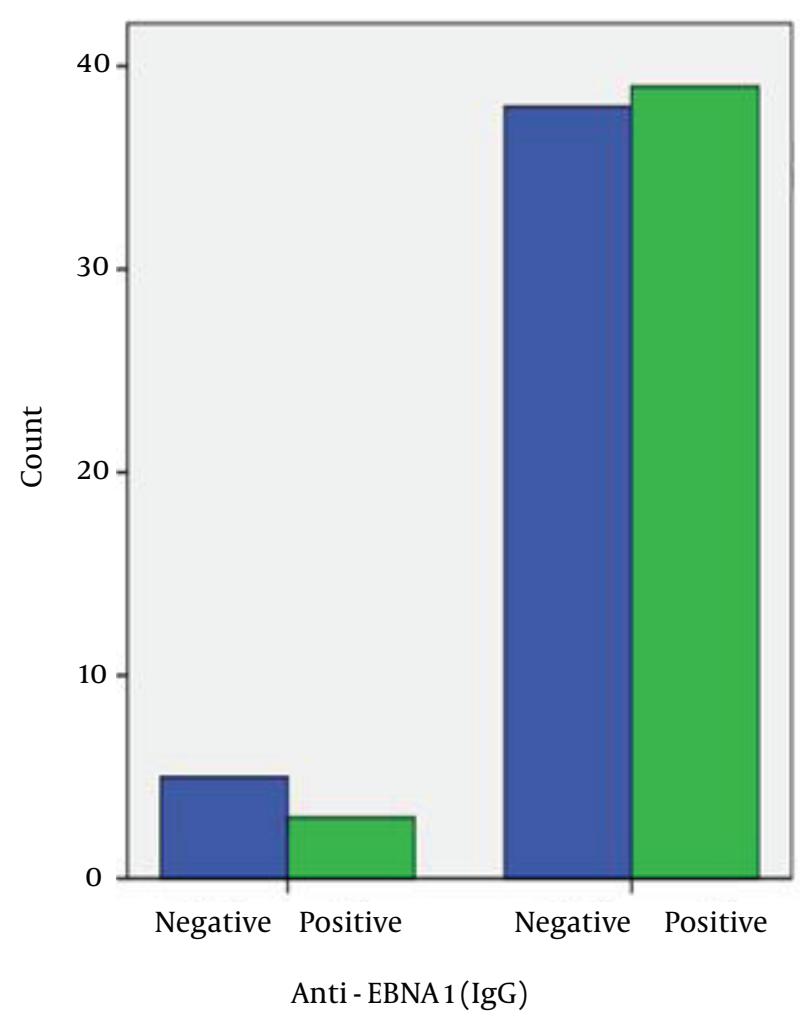

Figure 4. Bar Chart of Seropositivity to Anti-EBNA-1 IgG in the Patient (Green Bar) and Control (Blue Bar) Groups

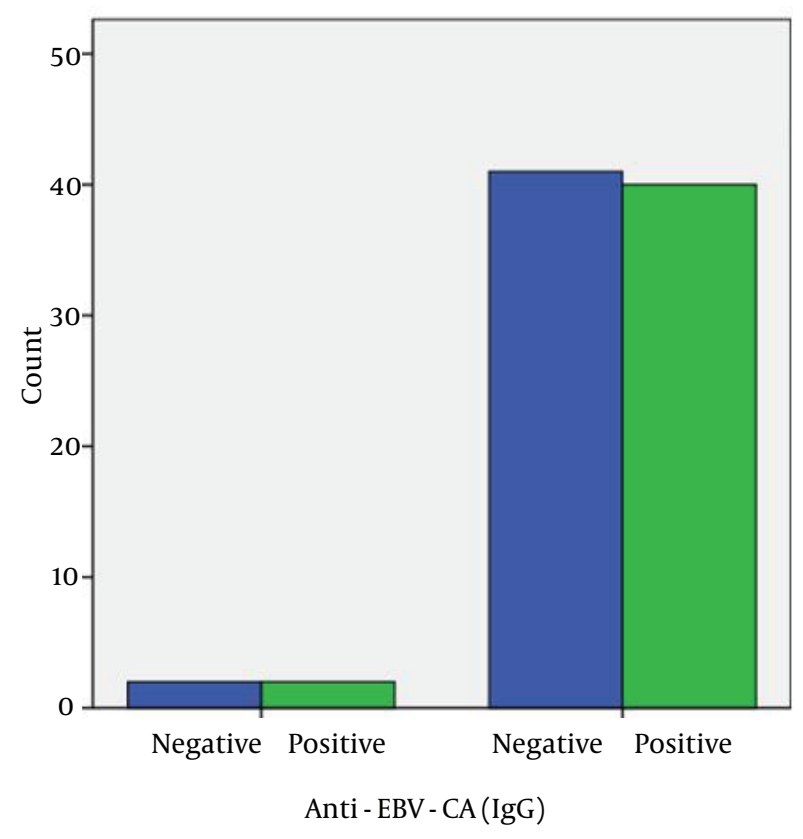

Figure 5. Bar Chart of Seropositivity to Anti-EBV-CA-IgG in the Patient (Green Bar) and Control (Blue Bar) Groups 
Honarmand $\mathrm{H}$ et al.

Table 1. Statistical Association between Seropositivity to Anti-EBNA-1 IgG, Anti-EBV-CA IgG, and Anti-EBV-EA-D IgG and the Occurrence of MS ${ }^{\text {a }}$

\begin{tabular}{lccccccc}
\hline \multirow{2}{*}{ ELISA tests } & \multicolumn{2}{c}{ Seropositivity Rate } & \multicolumn{4}{c}{ Statistical Analysis } \\
\cline { 2 - 8 } & Control & Patients & P1 & FET & CC & P2 & OR \\
\hline Anti-EBNA-1 IgG & $92.9 \%$ & $88.4 \%$ & 0.479 & 0.713 & 0.077 & 0.485 & 1.71 \\
Anti-EBV-CA IgG & $95.2 \%$ & $95.3 \%$ & 0.981 & 1.000 & -0.003 & 0.981 & 1.46 \\
Anti-EBV-EA-D IgG & 0 & $4.7 \%$ & 0.157 & 0.494 & -0.153 & 0.161 & - \\
Both Anti-EBNA-1 and Anti-EBV-CA(IgG) & $95.2 \%$ & $90.6 \%$ & 0.317 & 0.616 & 0.003 & 0.689 & 1.95 \\
\hline
\end{tabular}

a Abbreviations: CC, Spearman Rho Correlation Coefficient; FET, Fisher Exact Test Exact Sig. (2-Sided); MS, Multiple Sclerosis; OR, Odds Ratio; P1, Pearson Chi-Square Asymptotic Significance (2-Sided); P2, Spearman Rho Correlation Test Significance (2-Sided).

\begin{tabular}{|c|c|c|c|c|c|c|c|c|c|c|}
\hline \multirow[t]{2}{*}{ Parameters } & \multicolumn{4}{|c|}{ Anti-EBNA-1 IgG } & \multirow[t]{2}{*}{ Comment } & \multicolumn{4}{|c|}{ Anti-EBV-CA IgG } & \multirow[t]{2}{*}{ Comment } \\
\hline & P1 & LLA & CC & $\mathbf{P 2}$ & & P1 & LLA & CC & P2 & \\
\hline Duration of Disease & 0.897 & 0.982 & 0.022 & 0.897 & NS & 0.984 & 0.409 & 0.182 & 0.273 & NS \\
\hline Number of Crises & 0.221 & 0.608 & 0.061 & 0.716 & NS & 0.961 & 0.434 & 0.178 & 0.285 & NS \\
\hline EDSS & 0.876 & 0.429 & 0.129 & 0.435 & NS & 0.782 & 1.000 & -0.031 & 1.000 & NS \\
\hline Age of Patients & 0.189 & 0.178 & 0.227 & 0.182 & NS & 0.022 & 0.032 & 0.362 & 0.030 & NS \\
\hline Gender of Patients & 0.426 & 0.432 & -0.127 & 0.440 & NS & 0.579 & 0.584 & 0.089 & 0.591 & NS \\
\hline
\end{tabular}

a Abbreviations: CC, Spearman Rho Correlation Coefficient; EDSS, Expanded Disability Status Scale; LLA, Linear-by-Linear Association Asymptotic Significance (2-Sided); NS, Not Significant; P1, Pearson Chi-Square Asymptotic Significance (2-Sided); P2, Spearman Rho Correlation Test Significance (2-Sided).

(11) found nearly $100 \%$ seroprevalence of antibodies to EBV in patients with MS and reported that elevated EBV antibody titers years before the clinical onset of the disease and an increased risk of MS after symptomatic primary EBV infection (infectious mononucleosis) suggest an association between MS and a previous infection with EBV. Levin et al. (12) concluded that MS risk is extremely low among individuals not infected with EBV, but it increases sharply in the same individuals following EBV infection. Christensen et al. (3) also reported that MS patients have elevated anti-EBV antibody responses, both in the serum and the cerebrospinal fluid. Ruprecht et al. (11) reported that the notion of a persisting (possibly immunological) change caused during the acute phase of primary EBV infection and subsequently leading to permanently elevated MS risk appears compatible with several aspects of the association found between MS and EBV. Elsewhere in another study, Levin et al. (13) found an age-dependent relationship between EBV infection and the development of MS.

DeLorenze et al. (15) stated that the elevation in anti-EBV titers is probably an early event in the pathogenesis of MS and is unlikely to be the result of a specific immune dysregulation. Farrell et al. (8) found that the heightened immune response to EBV in MS is specifically related to EBNA-1 IgG, a marker of the latent phase of the virus. In the present study, we chose to screen seropositivity to EBNA-1 and EBV-CA because they are the most important indicators of an EBV past infection and also we chose serological investigation of EBV-EA-D because it is the main indicator of active infection. The strongest predictors of MS were serum levels of IgG antibodies to the viral capsid antigen (VCA) or the Epstein-Barr viral nuclear antigen (EBNA) complex in the study of Levin et al. (12). Haahr et al. (14) showed that the heightened immune response to EBV in MS is specifically related to EBNA-1 IgG, a marker of the latent phase of the virus. DeLorenze et al. (15) reported that the elevations in antibody titers to the EBNA complex and EBNA-1 among their MS cases first occurred between 15 and 20 years before the onset of symptoms and persisted thereafter.

We did not find a significant association between seropositivity to EBNA-1 IgG and the development of classic MS, and nor did we detect a significant association between seropositivity to EBV-CA IgG and the development of MS. In addition, we found that seropositivity to both EBNA-1 and EBV-CA, which could be a potent indicator of persistent inactive (past) infection, was not significantly associated with the development of classic MS. Our finding is not consistent with that in the previous relevant studies. This difference might be related to other environmental factors that might contribute to the development of MS such as geographic factors, socioeconomic status, prevalence rate of MS in the area, age at EBV infection acquisition, and predominant type of EBV infection in the studied area. 
The prevalence of MS is low, while the prevalence of EBV is very high in the area where we carried out the present study. Moreover, age at EBV infection acquisition is in early childhood (symptomless type) and mononucleosis is not a common type of EBV infection in the area. These are not in favor of the establishment of a potent association between EBV infection and MS. The low prevalence rate of MS indicates the low environmental load of the predisposing factors (other than infection) in the area. High spreads of EBV infection at lower ages have shown a reverse association with the development of MS in several studies. Primary infection during adolescence or adulthood often manifests as infectious mononucleosis, which has been associated with a two- to threefold rise in the risk of MS (18). Haahr et al. (14) showed that during or after puberty, EBV is transmitted to a major proportion of the population in an MS high-prevalence area and there is an association between late infection with EBV and an increased risk of developing MS.

Using individuals infected with EBV in early childhood as the reference, the risk is about tenfold less among EBVnegative individuals, and about two- to threefold greater among those infected with EBV later in life (as inferred from a history of mononucleosis); thus, there is at least a 20-fold increase in risk among individuals with a history of mononucleosis compared with those who are EBV negative, despite their sharing a similar "high hygiene" childhood environment (16). Cohen (2) remarked that the risk of MS is significantly increased among individuals with a history of infectious mononucleosis, a common manifestation of EBV infection in adolescence or adulthood, as compared with individuals without such a history (1). That is a finding that suggests later age at infection with EBV further increases the odds of the development of MS. Further prospective studies indicate a 2.8-time higher tendency for the development of MS after infectious mononucleosis (1).

As with all herpes viruses, EBV establishes a lifelong infection providing continuous stimulation to the immune system, and antibody titers to diagnostic EBV antigens in healthy subjects tend to remain constant over time. Furthermore, it is extremely unlikely that these data reflect an increase in EBV infection after the onset of MS because there is a conspicuous absence of recent EBV infection among individuals with MS (17). The fact that EBV infection is associated with a dramatic increase in MS risk has been known for many years inasmuch as EBV infects more than 95\% of the adult population. This high rate of infection results in a low power of individual studies attempting to establish an association and, perhaps most importantly, in the illogical conclusion that a virus infecting almost everyone cannot cause a relatively rare disease such as MS (16). On the other hand, other investigations have shown no significant association between EBV infection and MS. Lunemann et al. (9) presented the quantification of EBV viral loads in peripheral blood mononuclear cells by real-time polymerase chain reaction (PCR), which showed higher levels of EBV copy numbers in some patients with MS, although the overall difference in viral loads was not statistically significant compared with that in the healthy virus carriers.

We did not find a significant difference in the immune response to EBNA-1, EBV-CA, and EBV-EA-D, the viral proteins associated with EBV, between the patient and control groups. These responses (IgG) were not significantly increased in the MS patients compared with the unaffected individuals. We conclude that EBV past infection could not be a causative factor in the development of MS and nor is it a protective factor against classic MS. Further studies are needed to understand any mechanism by which immune responses to an EBV protein might contribute to MS.

\section{Acknowledgements}

This work was derived from a research project approved and sponsored by The Research and Technology Branch of Guilan University of Medical Sciences, Rasht, Iran. We thank Mrs. Rasouli for her assistance with the laboratory work.

\section{Authors' Contributions}

This work is collaboration between all the authors. Hamidreza Honarmand designed the study and statistical analysis, wrote the protocol, and wrote the first draft of the manuscript. Masoumeh Ahmadi Jalali Moghadam managed the analyses of the study and edited the manuscript. Hamidreza Hatamian and Ali Roudbary managed the literature searches. All the authors read and approved the final manuscript.

\section{Funding/Support}

Guilan University of Medical Sciences, Guilan, Iran was supported the study.

\section{References}

1. Pohl D. Epstein-Barr virus and multiple sclerosis. J Neurol Sci. 2009;286(1-2):62-4.

2. Cohen JI. Epstein-Barr virus infection. N Engl J Med. 2000; 343(7):481-92.

3. Christensen T. The role of EBV in MS pathogenesis. Int MS J. 2006;13(2):52-7.

4. Ascherio A, Munch M. Epstein-Barr Virus and Multiple Sclerosis. Epidemiol. 2000;11(2):220-4.

5. Bagert BA. Epstein-barr virus in multiple sclerosis. Curr Neurol Neurosci Rep. 2009;9(5):405-10.

6. Ascherio A, Munger KL, Lennette ET, Spiegelman D, Hernán MA, Olek MJ, et al. Epstein-Barr Virus Antibodies and Risk of Multiple Sclerosis. JAMA. 2001;286(24):3083.

7. Levin LI, Munger KL, Rubertone MV, Peck CA, Lennette ET, Spiegelman D, et al. Multiple sclerosis and Epstein-Barr virus. JAMA. 2003;289(12):1533-6.

8. Farrell RA, Antony D, Wall GR, Clark DA, Fisniku L, Swanton J, et al. Humoral immune response to EBV in multiple sclerosis is associated with disease activity on MRI. Neurology. 2009;73(1):32-8.

9. Lunemann JD, Edwards N, Muraro PA, Hayashi S, Cohen JI, Munz $C$, et al. Increased frequency and broadened specificity of latent EBV nuclear antigen-1-specific T cells in multiple sclerosis. Brain. 2006;129(Pt 6):1493-506. 
10. Ascherio A, Munger KL. Epstein-barr virus infection and multiple sclerosis: a review. J Neuroimmune Pharmacol. 2010;5(3):271-7.

11. Ruprecht K. [Multiple sclerosis and Epstein-Barr virus : new developments and perspectives]. Nervenarzt. 2008;79(4):399-407.

12. Levin LI, Munger KL, O'Reilly EJ, Falk KI, Ascherio A. Primary in fection with the Epstein-Barr virus and risk of multiple sclerosis. Ann Neurol. 2010;67(6):824-30.

13. Levin LI, Munger KL, Rubertone MV, Peck CA, Lennette ET, Spiegelman D, et al. Temporal relationship between elevation of epstein-barr virus antibody titers and initial onset of neurological symptoms in multiple sclerosis. JAMA. 2005;293(20):2496-500.

14. Haahr S, Plesner AM, Vestergaard BF, Hollsberg P. A role of late
Epstein-Barr virus infection in multiple sclerosis. Acta Neurol Scand. 2004;109(4):270-5.

15. DeLorenze GN, Munger KL, Lennette ET, Orentreich N, Vogelman JH, Ascherio A. Epstein-Barr virus and multiple sclerosis: evidence of association from a prospective study with long-term follow-up. Arch Neurol. 2006;63(6):839-44.

16. Ascherio A, Munger KL. Environmental risk factors for multiple sclerosis. Part I: the role of infection. Ann Neurol.2007;61(4):288-99.

17. Lennette ET, Rymo L, Yadav M, Masucci G, Merk K, Timar L, et al. Disease-related differences in antibody patterns against EBV-encoded nuclear antigens EBNA 1, EBNA 2 and EBNA 6. Eur J Cancer. 1993;29A(11):1584-9. 